\title{
IOFFE INSTITUTE GRB EXPERIMENTS: PAST, PRESENT AND FUTURE
}

\author{
R.L. Aptekar ${ }^{1}$, S.V. Golenetskii ${ }^{1}$, D.D. Frederiks ${ }^{1}$, E.P. Mazets ${ }^{1}$ \\ and V.D. Palshin ${ }^{1}$
}

\begin{abstract}
The short review of GRB studies performed for many years by Ioffe Institute is presented. An important breakthrough in GRB studies became possible owing to four Konus experiments carried out by the Ioffe Institute onboard the Venera 11 to 14 interplanetary missions from 1978 to 1983. The joint Russian-American Konus-Wind experiment, which has already been operating for more than 18 years, provides important and often unique data regarding GRB characteristics in $20 \mathrm{keV}-15 \mathrm{MeV}$ energy range. These investigations were complemented by several Konus and Helicon experiments onboard Russian near-Earth spacecraft. A short description of future Konus-UF and Konus-M experiments are also given.
\end{abstract}

\section{Introduction}

Cosmic gamma-ray bursts were discovered in 1967-1973 by the U.S. Vela satellites (Klebesadel et al. 1973). One of the first confirmations of the discovery of this new astrophysical phenomenon was provided by observations of the Ioffe Institute made on board Kosmos-461 satellite (Mazets et al. 1974). We present the results of many years' study of GRBs, performed by Ioffe Institute onboard interplanetary space missions and near-Earth satellites. Many of the basic characteristic of GRBs, such as their time profiles and energy spectra, and the first all-sky map of their source distribution on the celestial sphere, were determined using early Konus experiments onboard the Venera-11, -12, -13, and -14 deep space missions in 1978-1983. As a result of the observations of the giant flare on March 5, 1979, and a series of short repeated burst from its source the new class of rare astrophysical phenomena was discovered. In the somewhat later, these sources were named soft gamma repeaters (SGRs). In the subsequent Konus and Helicon experiments studies of GRBs and SGRs were continued, new SGR sources were found, and other

${ }^{1}$ Ioffe Physico-Technical Institute, St. Petersburg 194021, Russia 
SGR-related giant flares were also observed. Recently and most notable, Konus measurements have been central in finding SGR sources in the galaxies M81/M82 and M31, far outside our own Milky Way system. In the modern epoch of multiwave studies of cosmic gamma-ray bursts, the joint Russian-American Konus-Wind experiment, which has already been operating for more than 18 years, provides important and frequently unique data regarding temporal and spectral parameters of GRBs though the $20 \mathrm{keV}$ to $15 \mathrm{MeV}$ energy range. A short description of future Konus-UF and Konus-M experiments are also given.

\section{Study of GRBs from the Venera interplanetary missions in 1978-1983}

An important breakthrough in studies of GRBs was made possible owing to four Konus experiments carried out by the Ioffe Institute on board the Venera 11, 12, 13 \&14 deep space missions in 1978-1983. Each Konus instrument consisted of six scintillation detectors with close to cosine angular sensitivity; detectors were arranged along six axes of the spacecraft. It gave an opportunity to determine the direction towards GRB sources independently using single spacecraft data. The second approach to localize GRB source position in the Konus experiments was a triangulation method. The Venera interplanetary missions were launched in pairs and separated each from other by distance measured by several tens million kilometers. Such large distance between missions provided a high accuracy for GRB source localization on the celestial hemisphere. Therefore the Konus experiments had two independent methods for GRB source localization: the autonomous approach based on the data from detectors with an anisotropic angular sensitivity and the triangulation method using large distances between Venera missions.

The Konus observations of the temporal structures of GRBs had revealed for the first time the existence of a separate class of short bursts, demonstrating the so-called "bimodal" duration distribution (Mazets et al. 1981) (Fig. 1). It had been for the first time established that GRBs were randomly distributed over the celestial sphere (Mazets \& Golenetskii 1988) (Fig. 2).

Later, these findings were confirmed with an even larger number of events in the well-known BATSE experiment on board NASA's Compton Gamma-Ray Observatory (Paciesas W.S. et al. 1999).

Another key result of the Konus experiments onboard Venera missions came from the observations of the giant flare on March 5, 1979 (Fig. 3) and a series of short repeated burst from its source (Fig. 4). As a result, a new class of rare astrophysical phenomena was discovered (Mazets et al. 1979; Golenetskii et al. 1983), which somewhat later were named soft gamma-ray repeaters (SGRs).

\section{The Konus-Wind experiment}

A new and important chapter in the research of GRBs and SGRs carried out by the Ioffe Institute is associated with a joint Russian-American experiment which is being conducted using the Russian Konus scientific instrument onboard the 


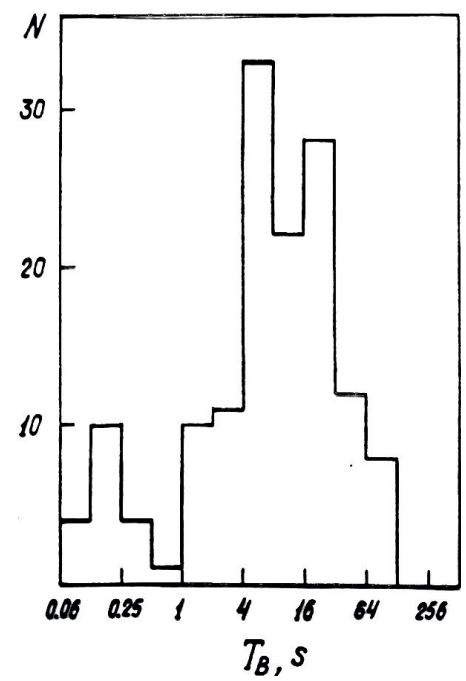

Fig. 1. Konus observations of the GRBs temporal structures revealed the existence of a separate class of short bursts (Mazets et al. 1981).

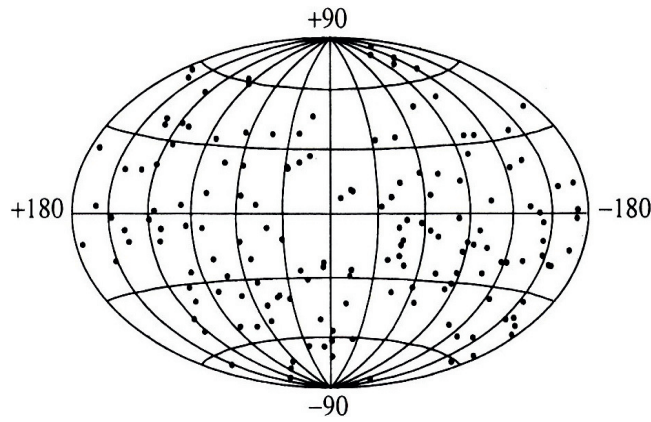

Fig. 2. Localizations of GRB sources demonstrated their isotropic distribution on the celestial sphere (Mazets \& Golenetskii 1988).

U.S. Wind spacecraft. The trajectory of the Wind spacecraft is maintained in the vicinity of the L1 Lagrangian point and the instrument has up to 5 light-seconds lag from the near-Earth GRB observing missions. Remote from the Earth and Moon, its exposure to entire celestial sphere is exceedingly favorable for studies of unpredictable and transient sources. Two high-sensitivity scintillation detectors of Konus-Wind gamma-spectrometer permanently observe the entire celestial sphere, so that no energetic event important to the astrophysics of GRBs and SGRs has yet been missed by the Konus-Wind experiment during all its 18 years of successful observations.

The Konus-Wind instrument is a scintillation gamma-ray spectrometer consisting of two identical spectrometric detectors of gamma-ray photons and the electronics unit (Aptekar et al. 1995). Each detector includes a $\mathrm{NaI}(\mathrm{Tl})$ crystal with a diameter of $130 \mathrm{~mm}$ and height of $75 \mathrm{~mm}$ placed in a thin-walled aluminum container with a beryllium entrance window. A highly-transparent lead-glass exit window is used to protect the detectors from the spacecraft background in the soft spectral region. Such detector provides a low energy threshold for recording radiation of 10-12 keV, the photon registration range up to $15 \mathrm{MeV}$ and the burst detection sensitivity at the $\sim 10^{-7} \mathrm{erg} \mathrm{cm}^{-2}$ level. These detectors have no analogue in the GRB observations with respect to the wide energy range and degree of protection from the spacecraft background. The detectors are located on the spacecraft stabilized by rotation in such a way that they constantly observe the northern and southern ecliptic hemisphere. 


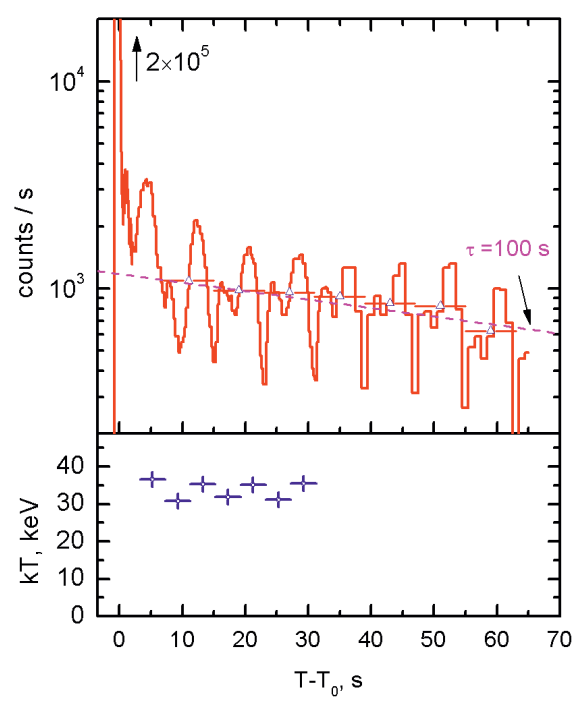

Fig. 3. Giant flare of SGR 0526-66 was observed on March 5, 1979 (Mazets et al. 1979).

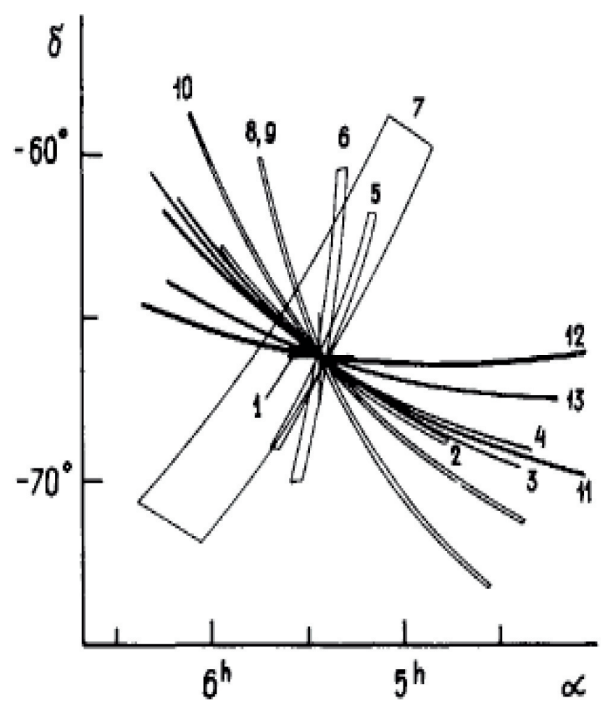

Fig. 4. Repeated bursts from SGR 0526-66 (Golenetskii et al. 1983).

The instrument operates in two distinct modes: the triggered burst detection and the background measurement. In the triggered mode, burst time profiles are recorded, in the current calibration, in the $20-80,80-300$, and $300-1200 \mathrm{keV}$ energy bands with the time resolution varying from $2 \mathrm{~ms}$ to $256 \mathrm{~ms}$ and total duration of registration lasting $\sim 230 \mathrm{~s}$. The standard program for recording an event time profile makes it possible to maintain a time resolution of $2 \mathrm{~ms}$ during the first $0.5 \mathrm{~s}$ of burst and for $0.5 \mathrm{~s}$ before burst. The instrument also has two special time analyzers (so called time "verniers"), which allow one to record with a high time resolution (2 ms) any light curve's section with sufficient increase in count rates. Two multichannel amplitude analyzers with quasi-logarithmic scales covering two energy intervals (20-1100 keV and $350 \mathrm{keV}-15 \mathrm{Mev})$ are used to measure event energy spectra. The accumulation time for each spectrum is automatically adjusted to the current burst intensity within a range from $64 \mathrm{~ms}$ to $8.192 \mathrm{~s}$.

The instrument calibrations and a method for reconstructing an initial gamma-ray spectrum from the instrumental spectrum recorded with Konus-Wind instrument is considered in (Terekhov et al. 1998). This technique was improved later by using a more advanced version of the program for calculating the detector response matrix (based on GEANT4) and the XSPEC12 package for modeling measured GRB spectra.

The possibility to continuously observe the entire celestial sphere and the wide energy range of observations in combination with the optimal program for recording GRB spectral characteristics are the main advantages of the Konus-Wind experiment. For this reason, the data of Konus-Wind experiment are widely used 
in present day multi-wave GRB observations. The Konus-Wind experiment is also an important vertex of the interplanetary network (IPN) of spacecraft with gamma-ray detectors, which localizes GRB sources by the triangulation method (for details see Pal'shin et al. 2013).

The famous "naked-eye" GRB 080319B studies illustrate the effective participation of the Konus-Wind experiment in multi-wave observations. The extraordinary optical brightness of this burst reached at a maximum $5.3 \mathrm{~m}$ (Racusin et al. 2008). The Konus-Wind instrument recorded detailed light curves of the bursts in the gamma-ray range from $18 \mathrm{keV}$ to $1160 \mathrm{keV}$; Figure 5 presents these measurements together with the data from two optical monitors.

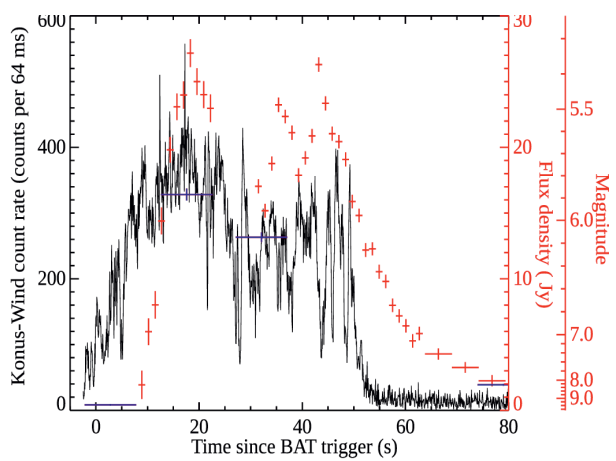

Fig. 5. Light curve of the GRB 090319B in the 18-1160 keV energy range according to the Konus-Wind experiments and optical monitors (Racusin et al. 2008).

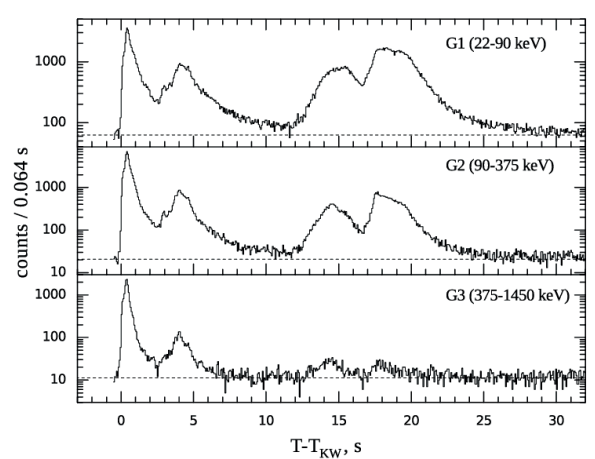

Fig. 6. The Konus-Wind light curves of the extremely bright GRB 110918A (Frederiks et al. 2013).

The gamma-ray light curve of this burst demonstrates multiple short spikes that do not correlate with the peaks in the optical range. Nevertheless, the gammarays and the optical emission of the burst source start and end at almost the same time, what evidently demonstrates that these radiations come from the same spatial region. The energy spectra of the GRB were studied in detail in the KonusWind experiment in the $20 \mathrm{keV}-7 \mathrm{MeV}$ range. These spectra demonstrate a strong spectral evolution in the form of pronounced radiation softening by the end of the main burst phase recorded in gamma-rays. According to the Konus-Wind data, this burst had a peak gamma-ray flux of $(3.26 \pm 0.21) \times 10^{-5} \mathrm{erg} \mathrm{cm}^{-2} \mathrm{~s}^{-1}$ in the $20 \mathrm{keV}-7 \mathrm{MeV}$ range, an integral energy flux of $(6.23 \pm 0.13) \times 10^{-4} \mathrm{erg} \mathrm{cm}^{-2}$ in the same range, and an isotropic equivalent energy release in gamma-rays of $1.3 \times 10^{54}$ erg (at $\mathrm{z}=0.937$ ) which places GRB 080319B among several gamma-ray bursts with the highest released energy.

One more GRB with an exceptional luminosity happened on September 18, 2011 was studied in detail in the Konus-Wind experiment. The long GRB 110918A was discovered by several GRB observing missions: INTEGRAL (SPI-ACS), Konus-WIND, Mars Odyssey (HEND), and MESSENGER (GRNS). This GRB was localized by the IPN and its bright X-ray counterpart was found close to 
the IPN box in the Swift/XRT follow-up observations starting 1.2 days after the trigger. The optical afterglow was discovered by the Isaac Newton Telescope and its spectroscopic redshift $\mathrm{z}=0.982$ was measured with the GMOS spectrograph mounted on the Gemini-N telescope (Levan et al. 2011). GRB 110918A is the brightest burst detected by Konus-WIND for more than 18 years of its continuous observations. The instrument's light curves in three energy bands covering the 22 - $1450 \mathrm{keV}$ range (Fig. 6) show an extremely bright, short, hard pulse followed by three weaker, softer, partly overlapping pulses within next 25 seconds. A spectral lag between the light-curves is determined, showing a substantial increase in the course of the burst. The emission is detected up to $12 \mathrm{MeV}$. Modeling the time-integrated energy spectrum with the Band function yields a moderate value of $\mathrm{E}_{\text {peak }}=340 \mathrm{keV}$, while the time-resolved spectral analysis reveals strong hardness-intensity correlation and a hard-to-soft evolution of the emission: $\mathrm{E}_{\text {peak }}$ falls from $\sim 2.3 \mathrm{MeV}$ at the onset of the huge initial pulse to $\sim 50 \mathrm{keV}$ at the final stage of the burst (Fig. 7). The total $20 \mathrm{keV}-10 \mathrm{MeV}$ energy fluence amounts to $(7.8 \pm 0.4) \times 10^{-4} \mathrm{erg} \mathrm{cm}^{-2}$ and a $64-\mathrm{ms}$ peak flux is $(9.2 \pm 0.4) \times 10^{-4} \mathrm{erg} \mathrm{cm} \mathrm{cm}^{-2} \mathrm{~s}^{-1}$, what corresponds to a huge isotropic-equivalent energy release of $(2.1 \pm 0.1) \times 10^{54} \mathrm{erg}$ and the record-breaking peak luminosity of $(4.7 \pm 0.2) \times 10^{54} \mathrm{erg} \mathrm{s}^{-1}$. The Konus-WIND results on GRB 110918A as well as the detailed coverage of the IPN localization, the X-ray afterglow observations with Swift/XRT, and the optical monitoring with Swift/UVOT can be found in the forthcoming paper (Frederiks, Hurley et al. in preparation).
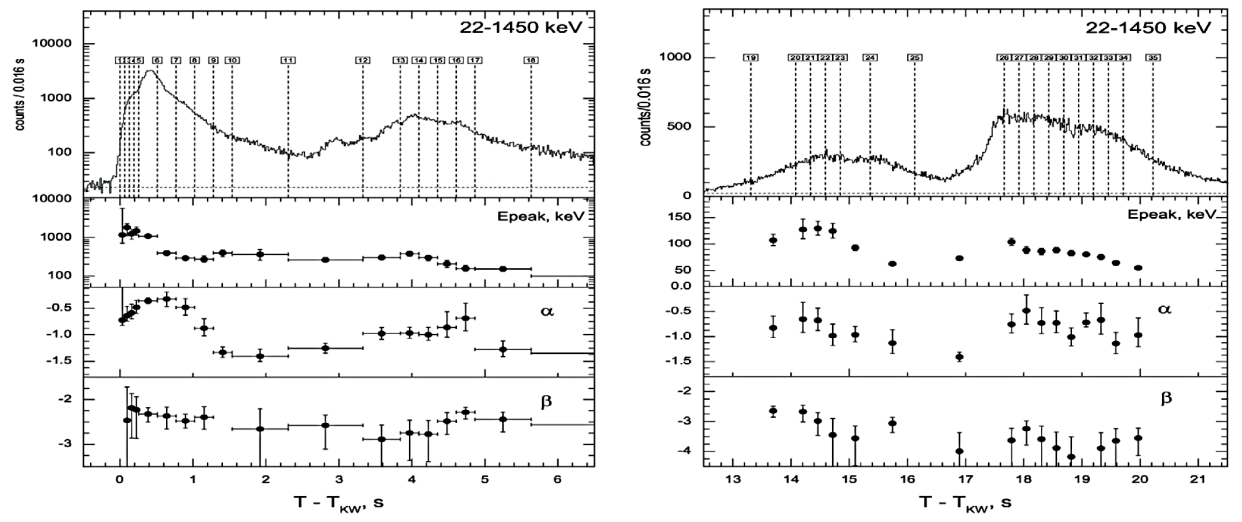

Fig. 7. Time-resolved spectral fits of GRB 110918A. The spectra are well described by the Band GRB function with the model parameters $\alpha, \beta$, and $\mathrm{E}_{\mathrm{p}}$ (Frederiks et al. 2013).

Thus, during the present-day epoch of intense multi-wave studies of cosmic gamma-ray bursts, the Konus-Wind experiment presents an important and often unique data on the time and energy properties of burst radiation in the $20 \mathrm{keV}-$ $15 \mathrm{MeV}$ range.

A very important and unusual result was obtained during simultaneous observations of the giant flare from SGR 1806-20 on December 27, 2004 by Konus-Wind 
and by another Ioffe Institute's instrument Helicon onboard Russian near-Earth solar space observatory CORONAS-F (Frederiks et al. 2007a). The photon counting rate of gamma-rays in the initial pulse of a giant SGR flare is always so great that sensitive detectors are fully overloaded ("saturated") in such a way that precise measurements of the initial pulse become difficult and only rough lower-bound estimates are possible. Positions of the spacecraft at the time of detection of this burst are shown schematically in Figure 8. The detectors of the Helicon instrument were screened by Earth from direct exposure to the initial pulse of the giant flare from gamma-repeater, but clearly recorded its reflection from the Moon's surface. This reduction in intensity allowed, for the first time, reliably reconstructing the temporal profile of the initial pulse of the giant flare (Fig. 6) and determining its energy parameters: the full isotropic energy release of $2.3 \times 10^{46} \mathrm{erg}$ and the peak luminosity of $3.5 \times 10^{47} \mathrm{erg} \mathrm{s}^{-1}[15]$. This detection of the 27 December 2004 giant flare became the first example of studying Moon-reflected X-ray and gamma-radiation coming from a source outside the Solar System.
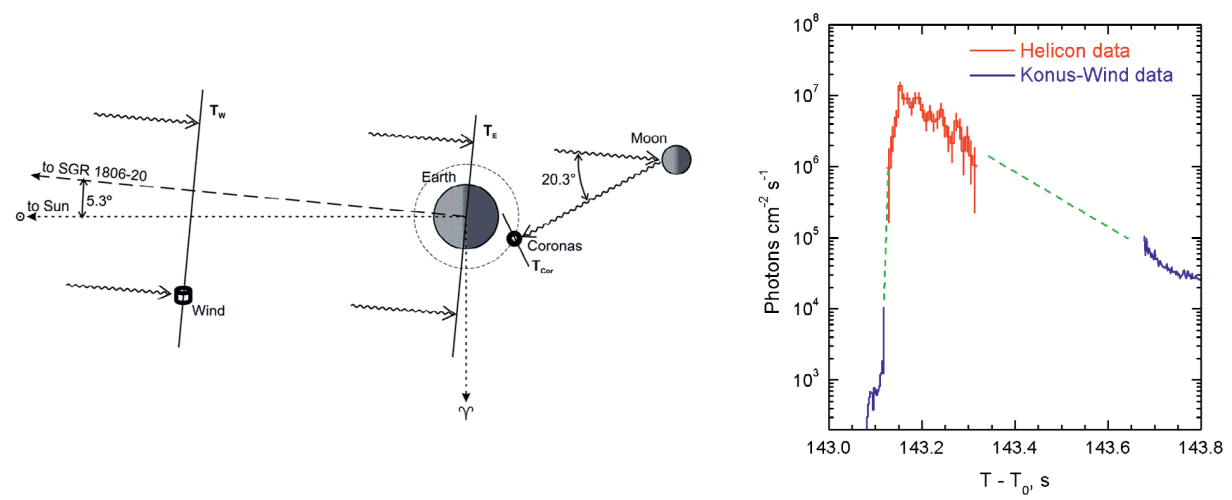

Fig. 8. Reflection of the initial pulse of the giant flare from SGR 1806-20 by the Moon and its detection by Konus-Wind and Helicon instruments (Frederiks et al. 2007a).

Recently, Konus-Wind measurements have been central in finding SGR sources in the galaxies M81/M82 and M31, first time far outside our own Milky Way system (Frederiks et al. 2007b; Mazets et al. 2008).

\section{The future loffe Institute experiments in the field of GRB study}

One of the future experiments is the Konus-UF instrument which is planned to install on board Russian Spectr-UF mission with the second name the World Space Observatory. The Konus-UF instrument consists of two detectors and an electronics unit. The Konus-UF detectors are built on $\mathrm{NaI}(\mathrm{Tl})$ scintillation crystals with dimensions like the Konus-Wind detectors have. The detectors will be allocated on the spacecraft body in such a manner as to observe a hemisphere (Fig. 9). The instrument is going to have a detailed program for measuring the temporal and 


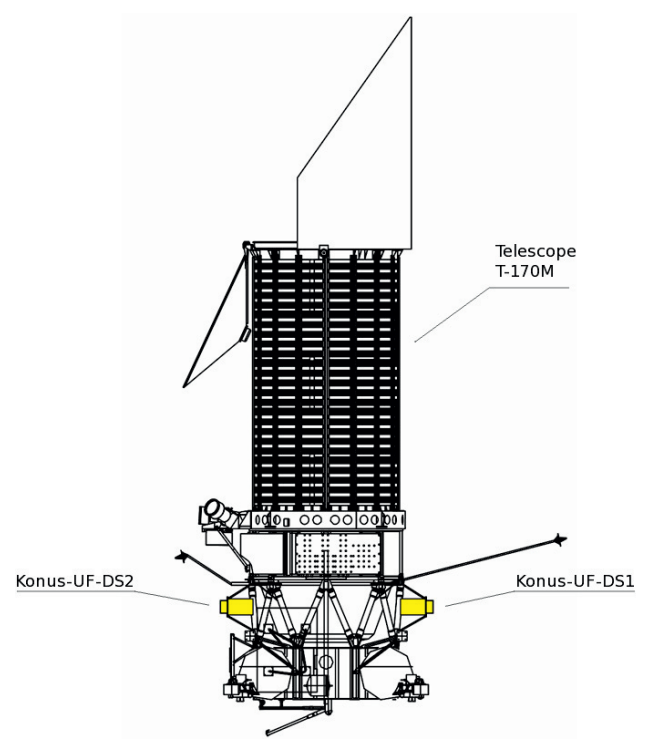

Fig. 9. Allocation of the Konus-UF detectors onboard the Spectrum-UF mission.

spectral characteristics of GRBs in the $10 \mathrm{keV}$ to $15 \mathrm{MeV}$ energy range. It is planned that the Spectr-UF mission is being launched in 2016 to a high-apogee orbit.

The second experiment is the Konus-M. It is planned that this experiment will be launched onboard a Small-size Scientific Spacecraft \#3 which is built by Lavochkin Assosiation in the frame of Program of small scientific spacecraft for fundamental space research. The spacecraft is planned to launch into a highapogee orbit by the end of 2014 .

Two sets of detectors are planed to use in the Konus-M experiment. The first one is a four-detector Konus-M-DN GRB localization system. Each Konus-M-DN detector includes a $\mathrm{NaI}(\mathrm{Tl})$ crystal with a diameter of $130 \mathrm{~mm}$ and a height of $15 \mathrm{~mm}$ in a thin-walled aluminum container with a beryllium entrance window and a high-transmission lead-glass exit window. These detectors have a passive shielding of their lateral surface and an anisotropic cosine-like angular sensitivity. Their placement on the spacecraft is shown in Figure 10. The axes of these four detectors are inclined to the spacecraft symmetry axis by $30^{\circ}$ and are separated in azimuth by $90^{\circ}$. Such system of Konus-M-DN detectors provides a GRBs source localization capability with an accuracy of $0.5-2.0$ degree depending on a burst intensity.

These detectors will observe a celestial hemisphere in the direction of $+\mathrm{X}$ of the spacecraft. Such a system of detectors was successfully used in the Ioffe Institute's Konus-A experiment on board Cosmos-2326 (Aptekar et al. 1998). The other set contains two spectrometric detectors (Konus-M-DS-1 and Konus-M-DS-2) which are similar to the Konus-Wind detectors. The axes of these detectors aligned 

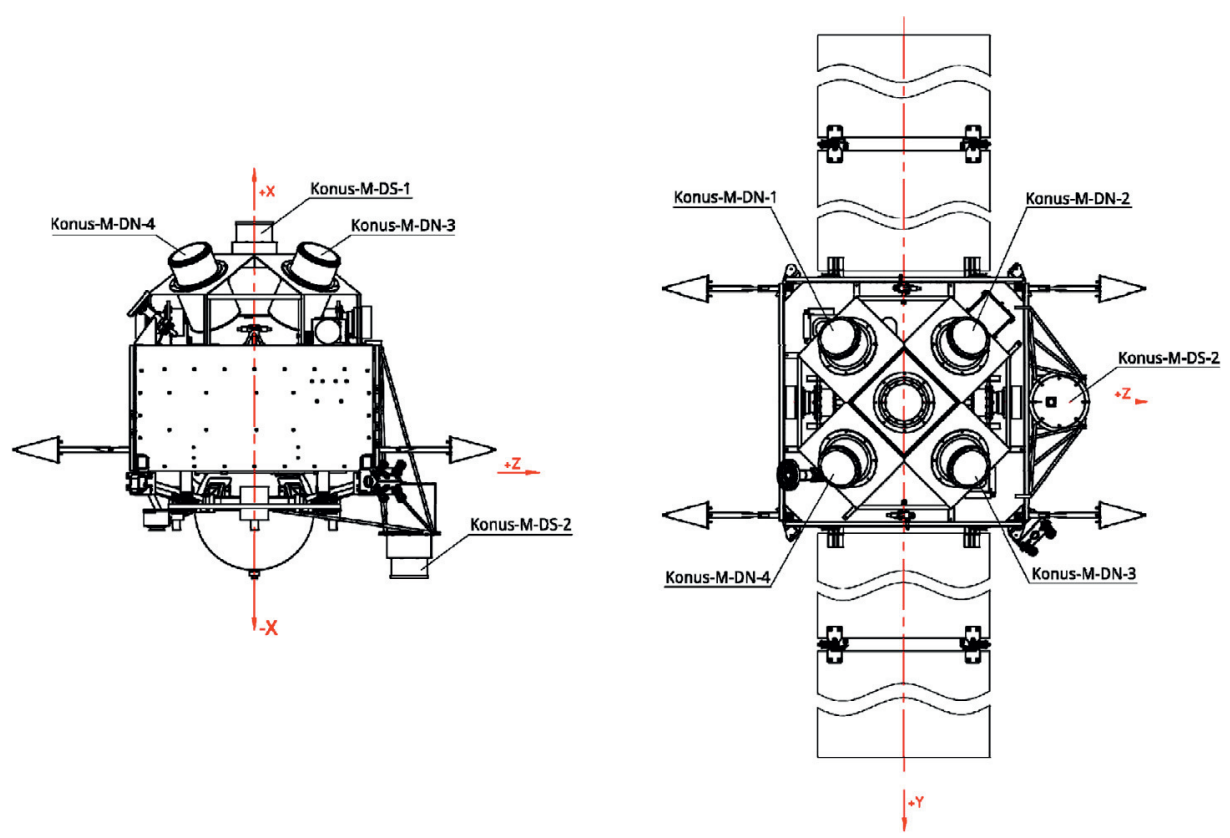

Fig. 10. The schematic arrangement of Konus-M detectors onboard spacecraft.

along the $+\mathrm{X}$ and $-\mathrm{X}$ axes of the spacecraft (Fig. 10). Thereby the Konus-M experiment will have a capability to constantly observe the whole celestial sphere and to obtain information about a GRB position simultaneously with temporal and spectral characteristics of bursts coming from a half of the sky.

\section{Conclusions}

The Ioffe Institute Konus experiments onboard Venera 11-14 interplanetary missions had for the first time investigated many of the basic characteristic of GRBs. The joint Russian-American Konus-Wind experiment, which has already been operated for more than 18 years, provides important and often unique data regarding the various characteristics of GRBs in the $20 \mathrm{keV}$ to $15 \mathrm{MeV}$ energy range. The future Ioffe Institute experiments will give us an opportunity to continue effective research of the extremely-explosive phenomena in the Universe.

Our work is supported by the state contract of the Federal Space Agency and Russian Foundation for Basic Research (projects numbers 11-02-12082-ofi-m and 12-02-000320-a).

\section{References}

Aptekar, R.L., Frederiks, D.D., Golenetskii, S.V., et al., 1995, Space Sci. Rev., 71, 265 Aptekar, R.L., Butterworth, P.S., Cline, T.L., et al., 1998, ApJ, 493, 404 
Frederiks, D.D., et al., this proceedings

Frederiks, D.D., Golenetskii, S.V., Palshin, V.D., et al., 2007a, Astron. Lett., 33, 1

Frederiks, D.D., Palshin, V.D., Aptekar, R.L., et al., 2007b, Astron. Lett., 33, 19

Golenetskii, S.V., Il'inkskii, V.N., \& Mazets, E.P., 1984, Nature, 307, 41

Klebesadel, R.W., Strong, I.B., \& Olson, R.A., 1973, ApJ, 182, L85

Levan, A.J., Tanvir, N.R., \& Wiersema, K., 2011, GCN Circular, 12368

Mazets, E.P., Golenetskii, S.V., \& Il'inskii, V.N., 1974, JETP Letters, 19,77

Mazets, E.P., Golenetskii, S.V., Il'inskii, V.N., Aptekar, R.L., \& Guryan, Yu. A., 1979, Nature, 282, 587

Mazets, E.P., Golenetskii, S.V., Il'inskii, V.N., et al., 1981, Ap\&SS, 80, 3

Mazets, E.P., \& Golenetskii S.V., 1988, Observations of cosmic gamma-ray bursts, Sov.

Sci. Rev., Sect. E, 6, 283

Mazets, E.P., Aptekar, R.L., Cline, T.L., et al., 2008, ApJ, 680, 545

Pal'shin, V.D., et al., 2013, in press

Racusin, J.L., Karpov, S.V., Sokolowski, M., et al., 2008, Nature, 455, 183

Terekhov, M.M., et al., 1998, AIP Conf. Proc., 428, 894 